\title{
Measurement and Monte Carlo Simulation of Hold-off Characteristics of Pseudo Spark Discharge Gaps
}

\author{
Member \\ Non-member \\ Yukihiko Morokuma \\ (Keio University) \\ Non-member \\ Yoshihisa Mochida \\ (keio University) \\ Member \\ Goji Ono \\ (Tobu Railway Co. Ltd.) \\ Yoshiharu Nakamura \\ (Keio University)
}

We measured hold-off voltages in typical pseudo spark discharge gaps for four different working gases: $\mathrm{He}, \mathrm{Ne}, \mathrm{Ar}$, and $\mathrm{N}_{2}$. The effect of the cathode hole diameter on the hold-off characteristics was also investigated. In order to understand the present observations, a simple Monte Carlo simulation of electron and ion motions in the pseudo spark gap was carried out in $\mathrm{He}$.

Keywords: Pseudo spark discharge, hold-off voltage, Monte Carlo simulation

\section{INTRODUCTION}

J. Christiansen found a novel discharge mode realized in very low pressure (about $10 \mathrm{~Pa}$ ) with a pair of coaxial electrodes, each of which has a central hole and an almost screened hollow behind it. ${ }^{(1)}$ Because the discharge has very high rate of current rise $(>50 \mathrm{~A} / \mathrm{ns})$ and a very thin and dense discharge path $(<1 \mathrm{~mm}$ diameter), it is expected as one of potential candidates for high-power plasma switches. The spark gap also generates confined and high-energy electron and ion beams through the anode and cathode ${ }^{(2)}$, respectively, and it is expected as a promising beam source for applications in material processing and high energy physics.

Because of the novelty and possible wide applications of the pseudo spark discharge there are many experimental and theoretical studies on this discharge mode. Many of the important works are included in the recently published monograph on the pseudo spark discharges ${ }^{(3)}$. And detailed numerical simulations of the initial stage of the discharges were carried out recently by Boeuf and Pitchford and they identified the importance of the hollow cathode effect on the onset of this discharge mode ${ }^{(4)}$.

The hold-off voltage is one of the most fundamental characteristics of the pseudo spark discharge but intensive results on this characteristics have been rare so far. In the present study the hold-off voltage in typical pseudo spark gaps for four different working gases, $\mathrm{He}, \mathrm{Ne}, \mathrm{Ar}$ and $\mathrm{N}_{2}$ were measured as a function of the working gas pressure. The measurements were repeated for two different electrode hole diameters, 5 and $10 \mathrm{~mm}$. The present measurements indicated that the hold-off voltage gradually decreased as the pressure increased as the left-hand side of the Paschen curve does. But the present results showed that there was a clear plateau in the left-hand branch. The measured hold-off characteristics in helium was compared with the estimated hold-off voltages using a Monte Carlo simulation of motion of electrons and positive ions in the present configuration of the pseudo spark gap. The present simulation cannot give the hold-off voltage at the pressure lower than about $100 \mathrm{~Pa}$ and that suggests that the further inclusion of collision processes between the fast neutrals and the background gas particles may be necessary, which are not included both in the present simulation and in that of Boeuf and Pitchford ${ }^{(4)}$.

\section{EXPERIMENTAL METHODS AND RESULTS}

The schematic diagram of the present spark gap is shown in Fig.1.

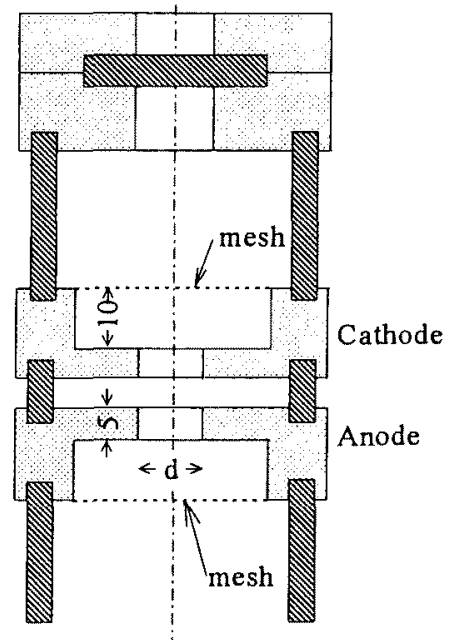

Fig.1. Schematic diagram of the pseudo spark gap.

The anode and cathode were, respectively, made of aluminum and Pyrex glass tube was used as the supporting insulators. The depth of the hollow behind each electrode is defined by mesh. The diameter and depth of the anode and cathode hollows are $30 \mathrm{~mm}$ and $10 \mathrm{~mm}$ respectively. The thickness of the anode and cathode is $5 \mathrm{~mm}$. The effect of the hole diameter, $\mathrm{d}$, on the hold-off voltage was examined at two different diameters, $5 \mathrm{~mm}$ and $10 \mathrm{~mm}$. The applied voltage from a stabilized high voltage source $(5 \mathrm{kV}$ max.) 
was fed through a $5 \mathrm{M} \Omega$ resistor and was increased slowly (1 volt/second) by using a PC. The hold-off voltage was determined as the applied voltage immediately before the voltage collapsed by a discharge. The variation of the holdoff voltage with repeating measurements was minimal. We measured the hold-off voltages for four different working gases: $\mathrm{He}, \mathrm{Ne}, \mathrm{Ar}$, and $\mathrm{N}_{2}$. The gases used are of the highest purity available. The base pressure of the vacuum system including the present spark gap was about $1 \times 10^{-5} \mathrm{~Pa}$ and degrading of the purity of the test gas by leakage during the measurement should be negligible.

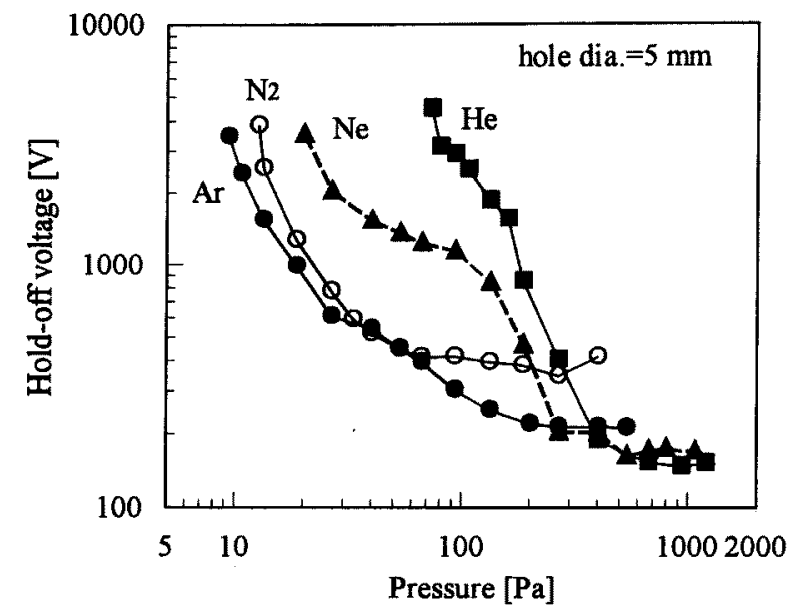

Fig. 2 The experimental hold-off voltage as a function of the working gas pressure. The diameter of the hole is $5 \mathrm{~mm}$.

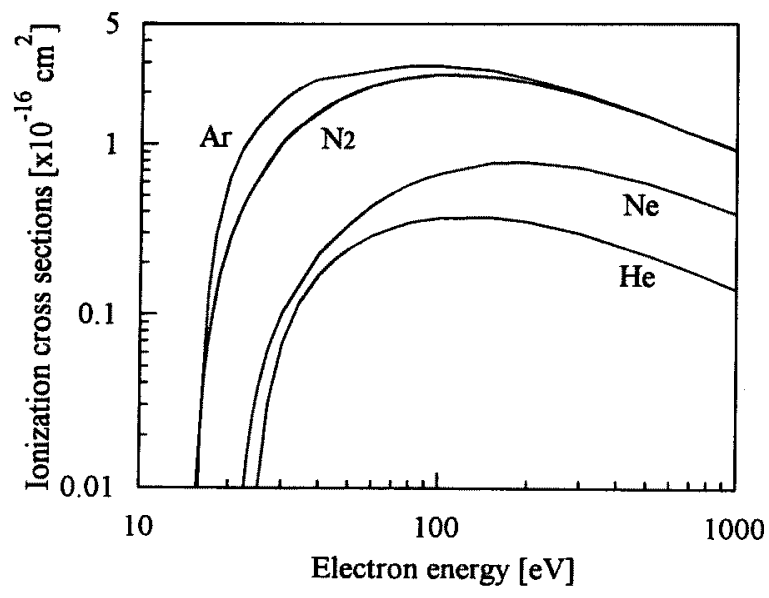

Fig. 3. The comparison of the ionization cross sections of He, $\mathrm{Ne}$, Ar and $\mathrm{N}_{2}$.

The experimental hold-off voltages as a function of pressure of working gas in the discharge gap with the $5 \mathrm{~cm}$ hole diameter are summarized in Fig. 2. The present results indicate that there are sharp transitions of breakdown voltage from the lower pressure to the higher pressure regions in rare gases (He, $\mathrm{Ne}$ and $\mathrm{Ar}$ ). In rare gases the hold-off voltage has a plateau in its left-hand branch. The plateau is apparent in $\mathrm{Ne}$ and $\mathrm{He}$ and is less apparent in $\mathrm{Ar}$. Independent experiment showed that the plateau was more apparent in two-fold gap. In nitrogen, however, the hold-off voltage depends on the gas pressure rather monotonously. The holdoff voltage in $\mathrm{He}$ was the highest among the gases investigated in low pressure region, and $\mathrm{Ar}$ and. $\mathrm{N}_{2}$ had similar hold-off voltages.

Fig. 3 shows the ionization cross sections for $\mathrm{He}, \mathrm{Ne}, \mathrm{Ar}$ and $\mathrm{N}_{2}$ by electron collisions ${ }^{(5)}$ and this figure explains the dependence of the hold-off voltage on the gas species in low pressures. The larger the ionization cross section in higher energy region $(>100 \mathrm{eV}$ ), the lower the hold-off voltage in low gas pressure.

The hold-off voltage in $\mathrm{N}_{2}$ is the highest in high pressure and this can be explained by the fact that the nitrogen molecule has dominant inelastic collision processes (rotationnal and vibrational excitations by electron impact) which can absorb electron energy effectively.

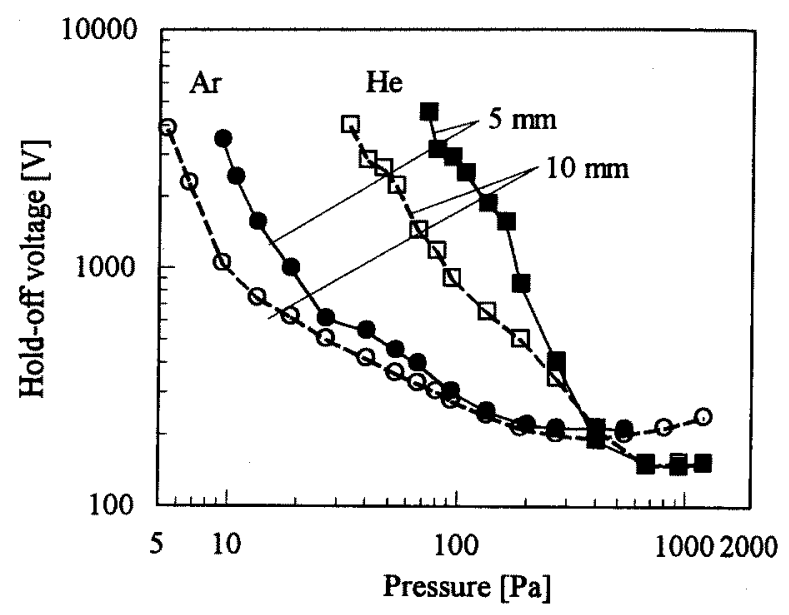

Fig. 4. The comparison of the hold-off voltages using different hole diameter. Solid and open symbols indicate the results using $5 \mathrm{~mm}$ - and $10 \mathrm{~mm}$-hole diameters, respectively.

In Fig. 4 shown are the hold-off voltages measured in the gaps having different hole diameters, $d=5$ and $10 \mathrm{~mm}$. The larger diameter allows the further penetration of the main gap electric field into the hollow, and that favors ionization multiplication and reduces the hold-off voltage ${ }^{(3)}$.

In the lower pressure region faint light emission was observed as the applied voltage approached to the hold-off voltage in all gases, and it is confined to the thin region $(<1$ $\mathrm{mm}$ ) along the electrode axis.

\section{MONTE CARLO SIMULATION AND RESULTS}

In order to understand the observations described above, a simple Monte Carlo simulation of electron and ion motions in the present pseudo spark discharge gap with the hole diameter $\mathrm{d}=5 \mathrm{~mm}$ in helium was carried out. Because of the working conditions of the pseudo spark gap (non-uniform electric field, low pressure, high voltage and short gap distance) it is natural to expect that electrons in the gap are in highly non-equilibrium condition and we have to be careful in assuming the local equilibrium approximation to analyze the phenomena. In these conditions the Monte Carlo method may be the most appropriate to the end. Since the space-charge should have little effects on the hold-off voltage, 
we can assume the static and space-charge free electric field.

The assumed electrode configuration was the same as used in the measurement (Fig. 5) and the potential at each 2D r-z grid point was calculated by the successive-over relaxation (SOR) method ${ }^{(6)}$. An appropriate number of initial electrons (typically 1000) were placed randomly in the cathode hollow and their 3D trajectories were followed after their starts with thermal speeds ( $\mathrm{T}=300 \mathrm{~K}$ was assumed).

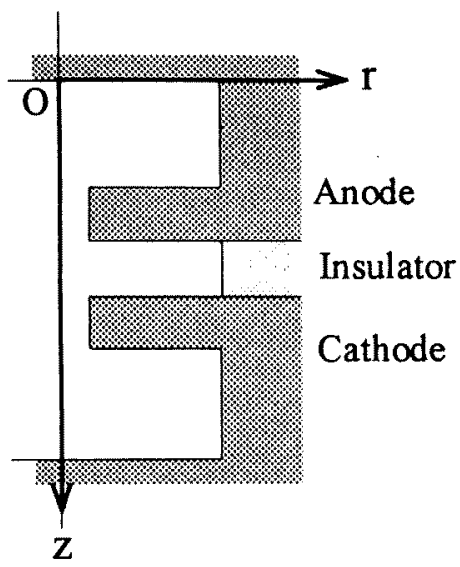

Fig. 5. Electrode configuration assumed in the present simulation.

The electric field acting on each electron at the start of each flight is assumed to be an weighted sum of fields of the four surrounding grid points in the $r-z$ plane ${ }^{(7)}$ and to be constant during one free flight in $\Delta t$, which corresponds to the collision probability $P=0.1$. If an electron passes more than one grid boundary during its flight, $P$ is reduced to one half and the trajectory calculation is repeated. After each flight an occurrence of collision, the type of collision if occurred and the new velocity are determined by generating uniform random numbers. All collisions are assumed isotropic. Electron collision processes of elastic momentum transfer $^{(8)}$, electronic excitation ${ }^{(9)}$ and ionization ${ }^{(5)}$ are

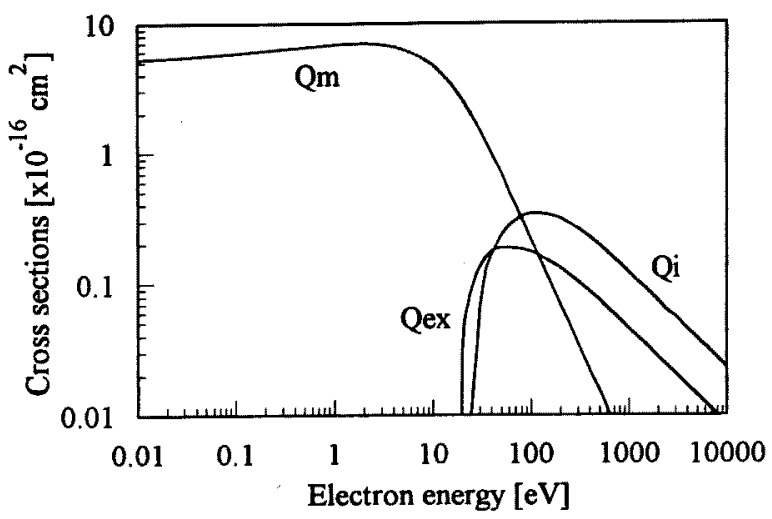

Fig. 6. Electron collision cross sections of the helium atom assumed in the present simulation.

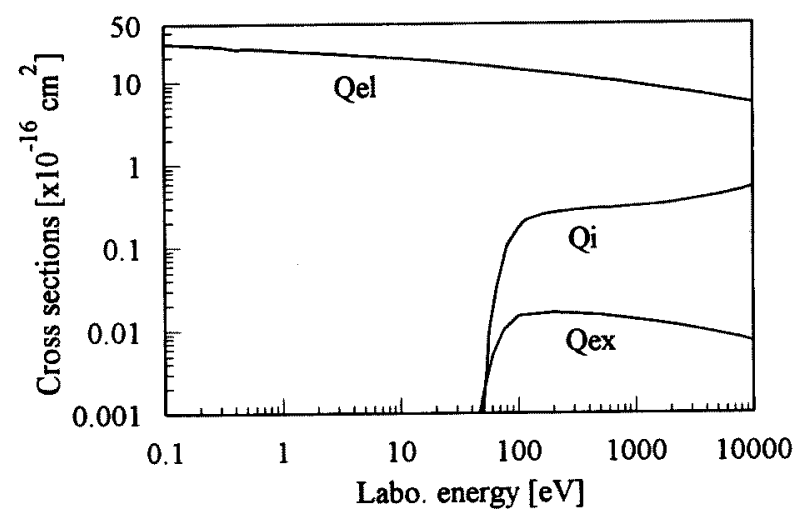

Fig. 7. $\mathrm{He}^{+}-\mathrm{He}$ collision cross sections assumed in the present simulation.

considered. The assumed set of the electron collision cross sections for the helium atom is summarized in Fig. 6 .

Positive ions produced in ionization collisions by electrons were also traced until they arrived at the cathode. Ion-neutral collision processes of charge transfer ${ }^{(10)}$, electronic excitation ${ }^{(10)}$ and collisional ionization ${ }^{(10)}$ with
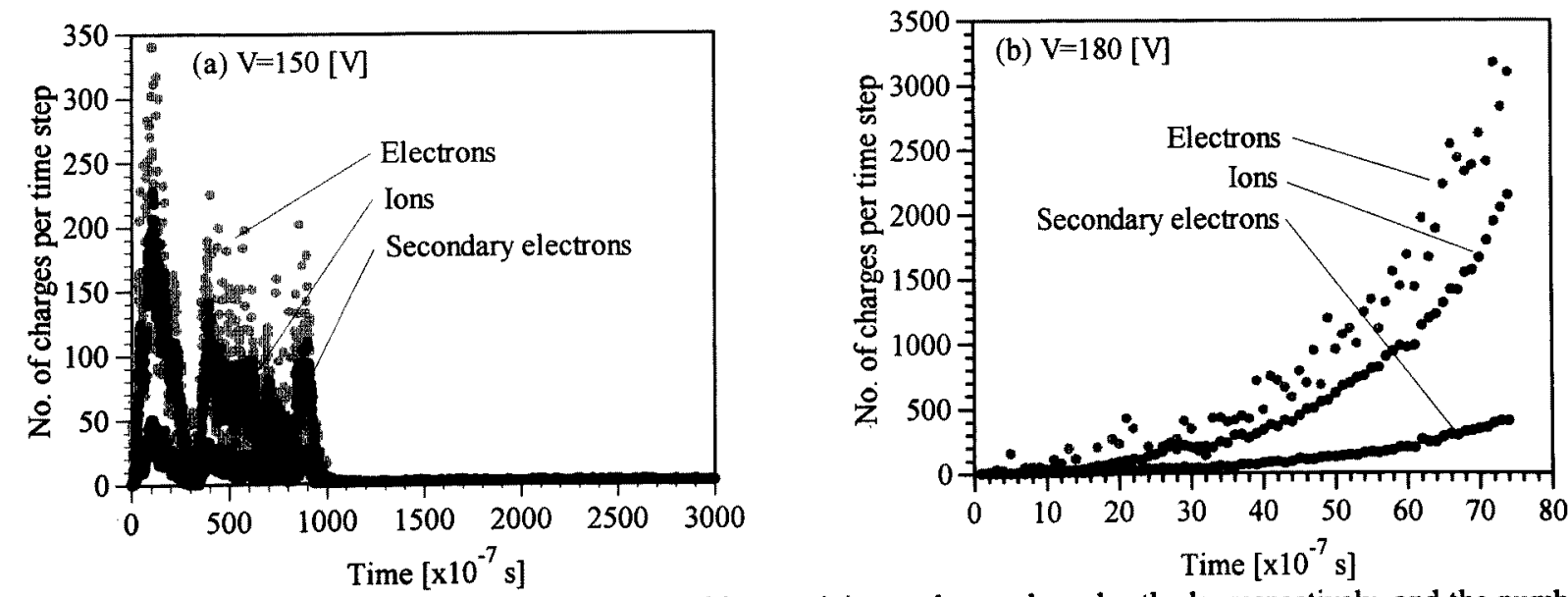

Fig. 8. Temporal behavior of the numbers of electrons and ions arriving at the anode and cathode, respectively, and the number of the secondary electrons emitted from the cathode. Helium pressure is $667 \mathrm{~Pa}$ and the sampling time step used in the present simulation is $10^{-7}$ second. (a) The applied voltage is 150 volts.

(b) The applied voltage is 180 volts. 

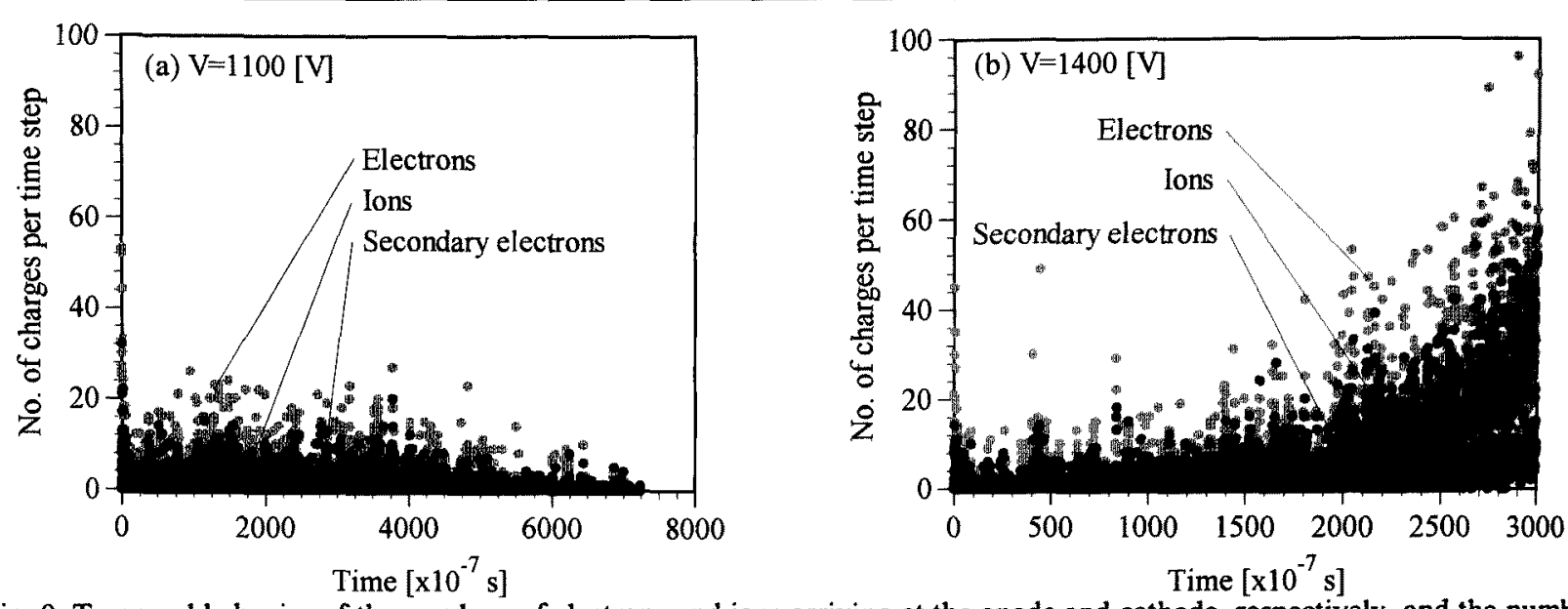

Fig. 9. Temporal behavior of the numbers of electrons and ions arriving at the anode and cathode, respectively, and the number of the secondary electrons emitted from the cathode. Helium pressure is $133 \mathrm{~Pa}$ and the sampling time step is $10^{-7}$ second.
(a) The applied voltage is 1100 volts.
(b) The applied voltage is 1400 volts.

neutral atoms were considered as well as the secondary electron emissions ${ }^{(11)}$ from the cathode. The cross section set of the $\mathrm{He}^{+}-\mathrm{He}$ collisions are summarized in Fig. 7. At each pressure the calculations were repeated by changing the main gap voltage in order to find the hold-off voltage in the following procedure. Each calculation was terminated either when all charged particles were absorbed in the electrodes (its maximum voltage is V1) or when the exponential growth with time of the number of the arriving charged particles at the cathode and anode was confirmed (its minimum voltage is V2) and the hold-off voltage was estimated to be found between the V1 and the V2.

Fig. 8 shows typical temporal behavior of the numbers of electrons and ions arriving at the anode and cathode, respectively, and the number of the secondary electrons emitted from the cathode by positive ions in helium at $667 \mathrm{~Pa}$ ( 5 Torr). The vertical scale indicates the number of charged particles at each sampling time step $\left(10^{-7}\right.$ second). At the applied voltage of $150 \mathrm{~V}$ (a) all electrons and ions in the gap

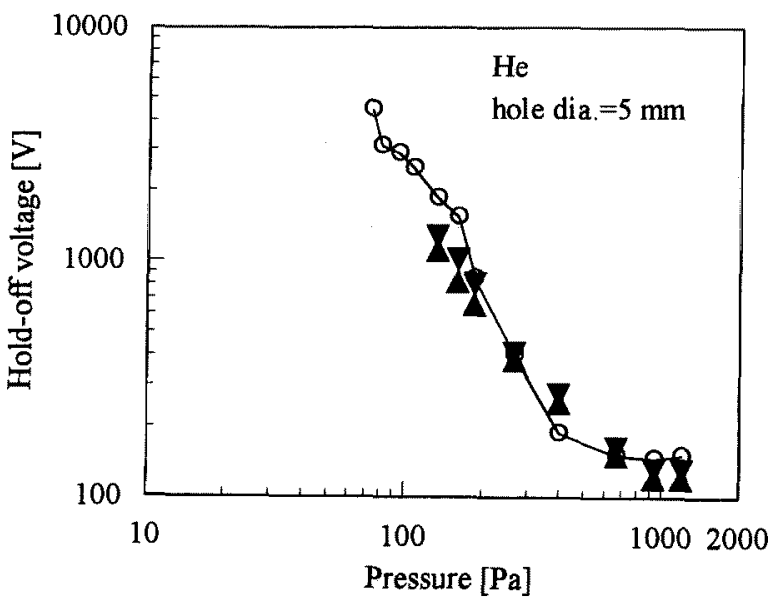

Fig. 10. Comparison of the hold-off voltages. Open circles show the experimental result. $\boldsymbol{A}$, simulated highest voltages when all charged particles are eventually swept to the electrodes (V1) ; $\mathbf{\nabla}$, simulated lowest voltages when exponential growth with time of the number of charged particles arriving at the cathode and anode is confirmed (V2). are absorbed by electrodes after several waves of avalanches, and at $180 \mathrm{~V}$ the number of ions and electrons increases with time exponentially (b). Fig. 9 shows the similar behavior in helium at low pressure $(133 \mathrm{~Pa})$. The temporal behavior of the charges in low pressure is much slower than that shown in Fig. 8 by orders of magnitude.

The V1 and V2 are compared with the measured holdoff voltage in Fig. 10. In the pressures higher than $200 \mathrm{~Pa}$ the present simulation can predict the measured hold-off voltage fairly well and that indicates the assumed collision processes are actually main mechanisms. In pressures lower than 100 $\mathrm{Pa}$, however, all charged particles are eventually absorbed by electrodes and exponential growth was not observed even at applied voltages higher than 10000 volts in the simulation.

This result indicates that additional charge multiplication processes must be included in low pressure ${ }^{(12)}$. Possible candidates are ionization collision between the fast neutrals generated by charge transfer collisions and the background gas particles and secondary electron emission by

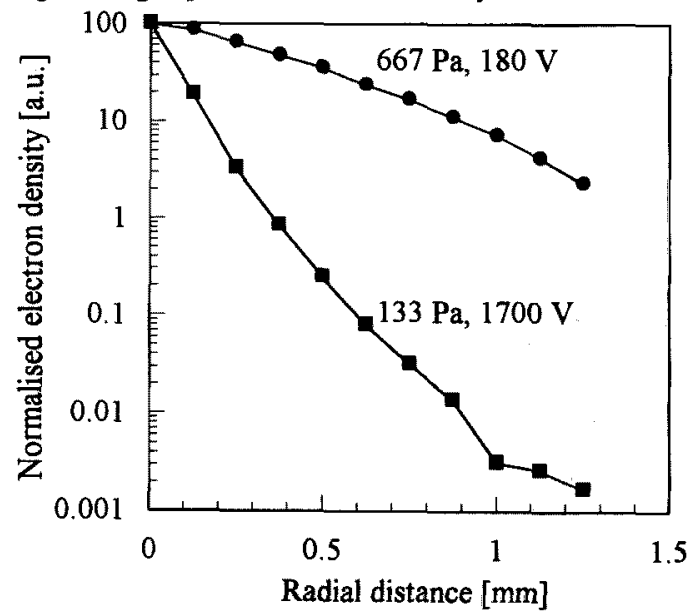

Fig.11. The normalized electron density distributions as a function of the radial distance. He pressure is $667 \mathrm{~Pa}$ and the main gap voltage is $180[\mathrm{~V}] ; \mathbf{E}$, He pressure is $133 \mathrm{~Pa}$ and the main gap voltage is 1700 [V]. 
the metastable particles generated by electron collisions and/or by the fast neutrals. The plateau observed in the lefthand branch of the hold-off voltage can be useful to identify working collision processes in pseudo spark discharge gap. We need further study on this point.

The time-integrated electron density distribution in low pressure $(133 \mathrm{~Pa})$ as a function of the radial distance is compared with that in high pressure $(667 \mathrm{~Pa})$ in Fig. 11. The distributions are normalized to 100 at the center. The density distribution in low pressure is confined in very thin region and the experimental observation of the thin light emission path is confirmed by the present simulation.

\section{CONCLUSIONS}

The hold-off voltages in typical pseudo spark discharge gaps with different hole diameter (5 and $10 \mathrm{~mm}$ ) were measured for four different working gases; $\mathrm{He}, \mathrm{Ne}, \mathrm{Ar}$, and N2. In rare gases the hold-off voltage has a plateau in its left hand branch. The dependence of the hold-off voltage on the gas species in low pressure should have a close relationship with their ionization cross sections in higher energy region.

The 3D Monte Carlo simulation of electrons and ions in the pseudo spark gap of the present experiment can predict the measured hold-off voltage fairly well in helium in the pressure range higher than $200 \mathrm{~Pa}$ and that indicate the assumed collision processes are actually working mechanism in the pressure range. The results of the present simulation in the pressure range lower than $100 \mathrm{~Pa}$, on the other hand, indicate that additional charge multiplication processes must be included in order to explain the observed experimental results.

\section{ACKNOWLEDGMENTS}

The present study was partly supported by the grant-inaid for promotion of scientific research programs, Keio University and by Inovative Research Institute (IRI).

(Manuscript received Pebruary 2, 1996, revised March 18, 1996)

\section{REFERENCES}

(1) J. Christiansen and C. Schultheiss: "Production of High Current Particle Beams by Low Pressure Spark Discharges", Z. Phys. A 290, 35-41 (1979)

(2) M. Ganciu, G. Modreanu, A. M. Pointu and I. I. Popescu, "Generation of intense pulsed electron beams by superposition of two discharges", J. Phys. D: Appl. Phys. 27, 1370-1374 (1994)

(3) M.A. Gundersen and G. Schaefer, eds.: "Physics and Applications of Pseudosparks", NATO ASI Series B: Physics Vol. 219 (1990 Plenum Press, New York)

(4) Jean-Pierre Boeuf and L.C. Pitchford: "Pseudospark Discharges Via Computer Simulation", IEEE Trans. Plasma Sci. 19, 286-296 (1991)

(5) D. Rapp and P. Englander-Golden, "Total Cross Sections for Ionization and Attachment in Gases by Electron Impact. I. Positive Ionization", J. Chem. Phys., 43, 1464-1479 (1965)
(6) R. W. Hockney and J. W. Eastwood, "Computer Simulation Using Particles", Chap. 6 (1988 Adam Hilger, Bristol)

(7) C. K. Birdsall and A. B. Langdon, "Plasma Physics Via Computer Simulation", Chap. 14 (1991 Adam Hilger, Bristol)

(8) M. Hayashi, "Recommended Values of Transport Cross Sections for Elastic Collision and Total Collision Cross Sections in Atomic and Molecular Gases", IPPJ-AM-19 (1981)

(9) H. S. W. Massey and E. H. S. Burhop, "Electronic and Ionic Impact Phenomena", Vol.1, Oxford University Press (1969)

(10)R. K. Janev et al, "Elementary Processes in HydrogenHelium Plasmas" (1987 Springer-Verlag, Berlin)

(11)P. C. Zalm and L. J. Beckers, "Secondary Electron Yields from Clean Polycrystalline Surface Bombarded by 5-20 keV Hydrogen or Noble Gas Ions", Philips J. Res. 39, 61-76 (1984)

(12) A.V. Phelps, "The application of scattering cross sections to ion flux models in discharge sheaths", J. Appl. Phys. 76, 747-753 (1994)

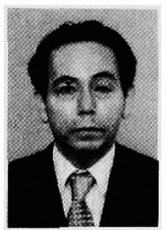

Yukihiko Morokuma (member) received B.E. and M.E. in Electrical Engineering from Keio University in 1965 and 1967. Since 1968, he has been with Keio University and is an Asistant Professor.

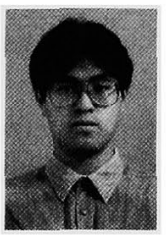

Yoshihisa Mochida (non-member) received B.E. in Electrical Enginnering from Keio University in 1995. He is studying in the graduate course of Keio University.

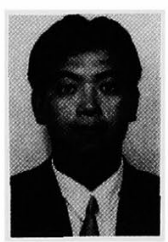

Goji Ono (non-member) received B.E. in Electrical Enginnering from Keio University in 1995. He is now working for Tobu Railway Co.Ltd.

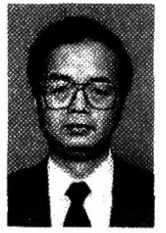

Yoshiharu Nakamura (member) received B.E., M.E. and Ph.D in Electrical Engineering from Keio University in 1966 , 1968 and 1976. Since 1969, he has been with Keio University and is an Associate Professor. 